\title{
Detection of virulence factors of South African Lactococcus garvieae isolated from rainbow trout, Oncorhynchus mykiss (Walbaum)
}

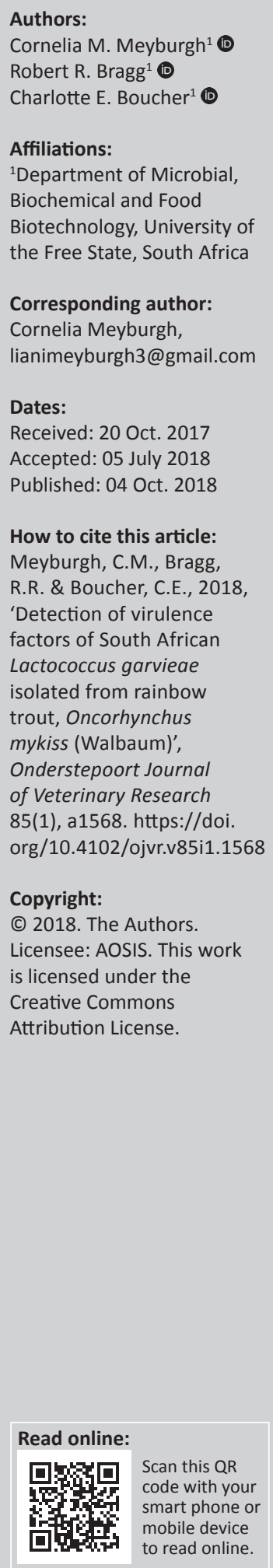

Lactococcus garvieae is a Gram-positive bacterium that causes mortalities in freshwater and marine fish worldwide and therefore results in severe economic losses in the aquaculture industry. Apart from the apparent integral role of the exopolysaccharide (EPS) capsule in pathogenesis, factors associated with virulence of this bacterium are poorly understood. However, recent studies have indicated that the ability of L. garvieae to cause disease does not depend on the presence of the EPS capsule. Lack of knowledge of virulence factors, pathogenesis and serology of L. garvieae is an impediment to the development of effective typing methods and control measures. This study, therefore, aimed to detect the presence of EPS capsules and other putative virulence factors in South African L. garvieae fish pathogenic isolates and a non-virulent isolate, and to identify possible candidates for subunit vaccine development. No indication of the presence of the EPS capsule was detected by negative staining or amplification of the EPS biosynthesis gene cluster in the virulent isolates or the avirulent strain, discrediting the notion that the EPS capsule is the sole determinant of virulence. However, a set of putative virulence factor genes was detected in all isolates, and candidates for subunit vaccine development (enolase, lactate dehydrogenase phosphoenolpyruvate-protein phosphotransferase) were identified by identification of extracellular proteins of virulent strains.

\section{Introduction}

Infectious disease caused by bacteria causes severe fiscal loss in aquaculture (Austin \& Austin 2012). Lactococcus garvieae, a pathogen of importance in the aquaculture of freshwater and marine fish (Bragg \& Broere 1986; Collins et al. 1983; Eldar et al. 1996; Kusuda et al. 1991), is described as a Gram-positive, facultative anaerobic, non-motile bacterium that does not produce endospores. It is generally described as an $\alpha$-haemolytic bacterium (Ravelo et al. 2001), but has been noted as $\beta$-haemolytic (Teixeira et al. 1996). A South African Gram-positive cocci isolate, initially described as Streptococcus spp. (Bragg \& Broere 1986), were reclassified as Enterococcus spp. and L. garvieae based on 16S ribosomal deoxyribonucleic acid DNA (rDNA) sequencing (Bekker et al. 2011). The bacterium is considered an emerging zoonotic agent, placing immunocompromised individuals at risk (Gibello et al. 2016).

Antimicrobial agents show strong in vitro activity against L. garvieae, but perform poorly under field conditions because of anorexia of infected fish (Bercovier et al. 1997) and possibly the ineffective metabolism of antibiotics in fish (Romero, Feijoó \& Navarrete 2012). Dissemination of antibiotic resistance in bacteria has grown into a global public health concern, accelerated by the unregulated and injudicious administration of antibiotics in humans and animals (Heuer et al. 2009). In aquaculture, chemotherapeutic treatment has led to the emergence of multiple resistance in streptococcal fish pathogens (Aoki et al. 1990; Austin \& Austin 2012). The spread of antibiotic resistance is aided by various mechanisms of horizontal gene transfer, of which plasmid-mediated transfer is the most widely documented in streptococcal fish pathogens. Vaccination is considered the best option to control lactococcosis because of the poor efficiency of chemotherapeutic agents under field conditions and the risks associated with the spread of antibiotic resistance determinants.

Bacteria employ a wide repertoire of virulence factors to promote survival within the host, some of which cause imminent damage to the host. Early studies on toxins of a non-Lancefield Streptococcus sp. (presumably L. garvieae) isolated from yellowtail showed the presence of a haemolytic toxin in culture supernatant (Kusuda \& Hamaguchi 1988). A study by Aguado-Urda et al. (2012) 
characterised five plasmids in a clinical isolate of L. garvieae strain 21881. The largest of these plasmids (68 $798 \mathrm{bp}$ [base pair]), pGL5, was shown to encode putative virulence factors, including a protein that posesses the enzymatic domain corresponding to the family of actin-ADP-ribosyltransferases (Aguado-Urda et al. 2012). Full genome sequencing of L. garvieae strains ATCC $49156^{\circledR}$ and Lg2 by Morita et al. (2011) identified several genes encoding putative virulence factors showing significant similarity to virulence factors of related species.

A comparative genome analysis of a virulent strain Lg2 and a non-virulent strain ATCC $^{\circledR} 49156$ of L. garvieae identified a 16.5 kilobase $(\mathrm{kb})$ capsule gene cluster, which is present in Lg2 but absent in ATCC $^{\circledast} 49156$ (Morita et al. 2011). The capsular gene cluster consists of 15 genes, of which 8 (eps-R, $X, A, B, C, D$ and cps- $L, W$ ) are conserved in the exopolysaccharide (EPS) biosynthesis gene cluster of four Lactococcus lactis strains isolated from human faecal samples (Morita et al. 2011). Analyses indicate that the capsular gene cluster is a genomic island, because of the presence of insertion sequences (ISs) on both ends of the capsular gene cluster. Even though the polysaccharide capsule is widely regarded as a major virulence factor of $L$. garvieae, it has been shown that non-capsulated strains Lgper and ATCC ${ }^{\circledR} 49156$ are pathogenic to rainbow trout, causing $89 \%$ and $98 \%$ mortality, respectively (Türe et al. 2014). These results suggest that the presence of the polysaccharide capsule cannot be directly correlated with pathogenicity in fish.

Despite the integral role of the polysaccharide capsule in pathogenesis, none has been structurally characterised. Understanding of the underlying genetic basis of variability in EPS structure and its likely interrelationship with serological variability in L. garvieae is lacking presently. Lack of knowledge of virulence factors, pathogenesis and serology of L. garvieae is an impediment to the development of effective typing methods and control measures. Therefore, this study aims to investigate the prevalence of various putative virulence factors in South African L. garvieae isolates and to speculate on their role in the process of infection in order to further elucidate the pathogenesis of lactococcosis in rainbow trout and identify possible targets for recombinant vaccine development.

\section{Materials and methods Isolates used in this study}

Cultures used in this study were obtained from a study by Bragg and Broere (1986) during which these bacteria were isolated from diseased rainbow trout, Oncorhynchus mykiss (Walbaum), in the former Eastern Transvaal (currently Mpumalanga) area and Johannesburg, Gauteng. Symptoms observed in diseased rainbow trout included extreme exophthalmos and rupture of one or both eyes, haemorrhaging of the ocular chamber, melanosis, enlargement of the spleen and haemorrhaging of intestines (Bragg \& Broere 1986). Geographic origins and isolate numbers (A1-A12), which will hereafter be used to refer to isolates, are presented in Table 1. Freeze-dried isolates were revived in brain heart infusion
TABLE 1: Isolate numbers and geographic origins of isolates used in the current study.

\begin{tabular}{lll}
\hline UFS number & Isolate number & Geographic origin \\
\hline UFSBC574 & A1 & TPA Lydenburg \\
UFSBC575 & A2 & Farm near Lydenburg \\
UFSBC576 & A3 & Farm near Lydenburg \\
UFSBC577 & A4 & Maloney's Eye - Johannesburg \\
UFSBC578 & A5 & G. Coetzee - Lydenburg \\
UFSBC579 & A6 & Lunsklip Fisheries - Lydenburg \\
UFSBC580 & A7 & Exact location unknown \\
UFSBC581 & A8 & Exact location unknown \\
UFSBC582 & A9 & Exact location unknown \\
UFSBC583 & A10 & Exact location unknown \\
UFSBC548 & A11 & Freshwater crab - Maloney's Eye \\
UFSBC547 & A12 & Dullstroom - Pleasant Ways trout farm \\
\hline
\end{tabular}

UFS, University of the Free State; TPA, Transvaal Provincial Administration.

(BHI) broth (Merck, 1.10493) and incubated under aerobic conditions at $30^{\circ} \mathrm{C}(\mathrm{A} 1-\mathrm{A} 3, \mathrm{~A} 5, \mathrm{~A} 6, \mathrm{~A} 11$ and $\mathrm{A} 12)$ for 48 hours or anaerobic conditions at $37^{\circ} \mathrm{C}$ (A4, A7-A10) for $48 \mathrm{~h}$ using an anaerobic jar with a gas-generating kit (Oxoid ${ }^{\mathrm{TM}}$ BR0038, Basingstoke, United Kingdom). Culture purity was confirmed by Gram staining. Revived isolates were stored in commercially available cryogenic vials (Microbank ${ }^{\mathrm{TM}}$, Pro-Lab Diagnostics, Toronto, Canada) at $-20^{\circ} \mathrm{C}$.

\section{Identification of isolates by 165 ribosomal DNA sequencing}

Isolates were cultivated in tryptic soy broth (TSB) (Merck, 1.05459) under aerobic conditions at either $30^{\circ} \mathrm{C}$ or $37^{\circ} \mathrm{C}$ for $18 \mathrm{~h}$ with no agitation. DNA was extracted according to the method described by Thanh (2006). Universal sequence primers 8F (5'-AGAGTTTGATCCTGGCTCAG-3') and 1525R (5'-AAGGAGGTGATCCAGCC-3') were used to amplify a 1517 base pair (bp) sequence of the 16S rDNA gene by polymerase chain reaction (PCR) (Beumer \& Robinson 2005). Amplicons were visualised by gel electrophoresis on a $1 \%$ agarose gel (Sambrook \& Russell 2001). For purposes of purification of the PCR product, the PCR products were run on a $2 \%$ weight per volume $(\mathrm{w} / \mathrm{v})$ low-melt agarose $\left(\mathrm{NuSieve} \mathrm{e}^{\circledR}\right.$ GTG $^{\circledast}$ Agarose). Bands were excised under ultraviolet illumination. The PCR products were purified from gel slices using a Wizard ${ }^{\circledR}$ SV Gel and PCR Clean-up System (Promega, Madison, WI). Sequencing reactions were prepared using Applied Biosystems ${ }^{\mathrm{TM}}$ BigDye $^{\circledR}$ terminator v.3.1 sequencing kit. Samples were submitted for Sanger sequencing at an inhouse facility at the University of the Free State. Sequence data were viewed using Geneious version 9 (http://www. geneious.com, Kearse et al. 2012) and sequences were used to query the National Centre for Biotechnology Information (NCBI) database using the nucleotide-nucleotide BLAST (BLASTn) algorithm (Altschul et al. 1990).

\section{Phenotypic characterisation of exopolysaccharides}

Negative staining with nigrosine was performed on isolates A1-A12 to determine the presence of EPS capsules. Pseudomonas aeruginosa and an avirulent reference strain of L. garvieae (NCFB657) were included as positive and negative controls, respectively. 


\section{Genotypic characterisation of exopolysaccharides}

The L. garvieae EPS synthesis gene cluster, as described in L. garvieae Lg2 (Miyauchi et al. 2012), was amplified in isolates A1-A3, A5, A6, A11 and A12 using the TaKaRa LA PCR Kit (TaKaRa Bio Inc. \#RR002A, Kyoto, Japan). Amplicons were visualised on a $1 \%$ agarose gel.

\section{Detection of putative virulence factor genes by polymerase chain reaction}

The presence of putative virulence factor genes, as identified by Morita et al. (2011), in South African isolates were investigated by PCR using primers designed by Türe and Altinok (2016). The presence of the following genes was investigated: haemolysins 1, 2 and 3 (hly1, -2, -3), NADH oxidase (nox), superoxide dismutase (sod), pneumococcal adherence and virulence factor $\mathrm{A}(p a v A)$ and pneumococcal surface adhesin A $(p s a A)$ (Table 2). Amplicons were visualised on a 1\% agarose gel. Products were purified using a Wizard ${ }^{\circledR}$ SV Gel and PCR Clean-up System (Promega, USA). Sequencing was performed using the Applied Biosystems ${ }^{\text {TM }}$ BigDye $^{\circledR}$ terminator v.3.1 sequencing kit. Obtained sequences were viewed and edited with Geneious 9 (Kearse et al. 2012) and used to query the NCBI database using the BLASTn algorithm (Altschul et al. 1990).

\section{Detection of extracellular virulence factors}

Lactococcus garvieae strains A1-A3, A5, A6, A11, A12 and NCFB657 were cultivated in TSB media for $24 \mathrm{~h}$ at room temperature. A negative control, consisting of sterile TSB, was included. Cells were pelleted by centrifugation at $20000 \times g$ for 2 minutes, and the supernatants filtered with a $0.2 \mu \mathrm{m}$ syringe filter unit to remove residual cellular components. Amicon ${ }^{\circledast}$ Ultra-4 10K Centrifugal Filter Units (Merck) with a molecular weight cut-off (MWCO) of $10 \mathrm{kDa}$ were used to concentrate extracellular proteins. A volume of $4 \mathrm{~mL}$ filtered supernatant was applied to the centrifugal filter and the unit was centrifuged at $7197 \times g$ for $20 \mathrm{~min}$. The eluate was discarded, the centrifugal filter was inverted inside a $50 \mathrm{~mL}$ Falcon tube and again centrifuged at $7197 \times g$ for $20 \mathrm{~min}$ to collect the concentrated protein fraction. Extracellular proteins, concentrated by Amicon ${ }^{\circledR}$ Ultra-4 10K Centrifugal Filter Units (Merck), were visualised by sodium dodecyl sulphate-polyacrylamide gel electrophoresis (SDS-PAGE). The polyacrylamide gel was stained and destained using the rapid Fairbanks Coomassie Blue protein staining and destaining method (Wong et al. 2000).

\section{Protein identification}

Prepared samples were submitted to the Facility for Genomics and Proteomics at the University of the Free State for identification by nanoscale liquid chromatography coupled to tandem MS (Nano LC-MS/MS) and by querying the SWISS-PROT annotated protein sequence database (Bateman et al. 2015) using the Mascot search engine that uses a probability-based scoring algorithm based on the molecular weight search (MOWSE) algorithm (Perkins et al. 1999).

\section{Results \\ Identification of isolates}

Amplification of the $16 \mathrm{~S}$ rDNA gene fragment by PCR yielded the expected \pm 1500 bp product, visualised by gel electrophoresis in Figure 1. The nucleotide BLAST search results, tabulated in Table 3, identified isolates A1-A3, A6, A11 and A12 as L. garvieae, A4 as Enterococcus faecalis, A7 as E. durans and A8-A10 as E. faecium. These results are consistent with the results of Bekker et al. (2011), who also identified this set of 12 isolates as 5 Enterococcus spp. and 7 L. garvieae isolates using $16 \mathrm{~S}$ rDNA sequencing (Bekker et al. 2011). No reports of fish-pathogenic Enterococcus spp. have been published; therefore, isolates A4 and A7-A10 were excluded from further experiments in this study.

\section{Phenotypic characterisation of exopolysaccharides}

\section{Negative staining}

As shown in Figure 2, no halos were observed in any of the isolates including the negative control NCFB657, in comparison with the positive control $P$. aeruginosa, where

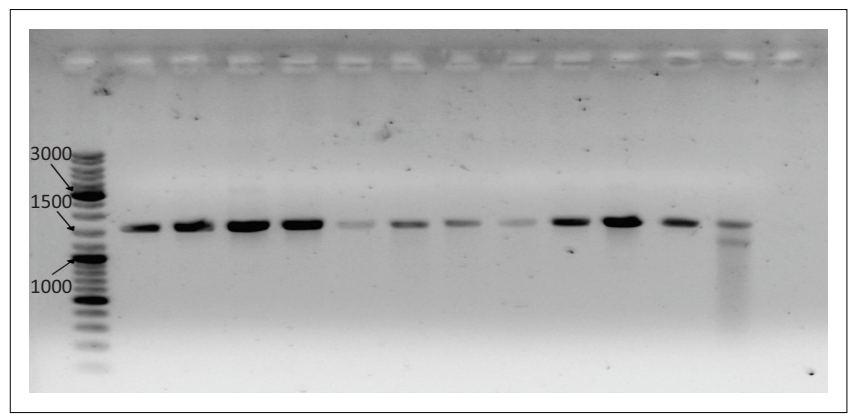

Note: Fragment sizes are indicated in bp (base pair)

FIGURE 1: Visualisation of $16 \mathrm{~S}$ polymerase chain reaction products of isolates $\mathrm{A} 1-\mathrm{A} 12$ on a $1 \%$ weight per volume $(\mathrm{w} / \mathrm{v})$ agarose gel. Bands in the expected size range $( \pm 1500 \mathrm{bp})$ were observed.

TABLE 2: Genetic loci and predicted gene products of candidate virulence genes, previously identified in Lactococcus garvieae Lg2 (Morita et al. 2011 ), under investigation in this study.

\begin{tabular}{llclll}
\hline Locus & Predicted gene product & Identity (\%) & Species & Accession number & Features \\
\hline LCGL_0323 & Haemolysin & 56 & Enterococcus faecalis & AAO81463 & Motif (PF03006) conserved in proteins with haemolytic activity \\
LCGL_0597 & Haemolysin & 72 & Streptococcus suis & EEF64743 & - \\
LCGL_0374 & Haemolysin & 59 & S. pyogenes & AAK33420 & Cleavable N-terminal signal sequence \\
LCGL_0664 & NADH oxidase (SP1469) & 51 & S. pneumoniae TIGR4 & - & - \\
LCGL_0285 & Superoxide dismutase (SP0766) & 68 & S. pneumoniae TIGR4 & - & - \\
LCGL_1330 & PavA (SP0966) & 62 & S. pneumoniae TIGR4 & - & Fibronectin-binding motif (PF05833) \\
\hline LCGL_1533 & PsaA (SP1650) & 49 & S. pneumoniae TIGR4 & - & - \\
\hline
\end{tabular}

Source: Bekker, A., Hugo, C., Albertyn, J., Boucher, C.E. \& Bragg, R.R., 2011, 'Pathogenic Gram-positive cocci in South African rainbow trout, Oncorhynchus mykiss (Walbaum)', Journal of Fish Diseases 34(6), 483-487. https://doi.org/10.1111/j.1365-2761.2011.01259.x 
TABLE 3: Top Basic Local Alignment Search Tool search hits generated for $16 \mathrm{~S}$ ribosomal DNA genes amplified from isolates A1-A12.

\begin{tabular}{|c|c|c|c|c|c|c|}
\hline Isolate & Nucleotide BLAST result & Query length & Query cover (\%) & E value & Identity (\%) & Accession $\mathrm{nr}$ \\
\hline A1 & Lactococcus garvieae strain A4 16S ribosomal RNA gene, partial sequence & 619 & 100 & 0.0 & 100 & KT924257.1 \\
\hline A2 & Lactococcus garvieae strain A2 $16 \mathrm{~S}$ ribosomal RNA gene, partial sequence & 783 & 100 & 0.0 & 100 & КT924256.1 \\
\hline A3 & Lactococcus garvieae strain RCB99 $16 \mathrm{~S}$ ribosomal RNA gene, partial sequence & 742 & 100 & 0.0 & 100 & KT260311.1 \\
\hline A4 & Enterococcus faecalis strain RCB986 $16 \mathrm{~S}$ ribosomal RNA gene, partial sequence & 466 & 100 & 0.0 & 99 & KT261198.1 \\
\hline A5 & Lactococcus sp. SI-Km(R)-3B $16 \mathrm{~S}$ ribosomal RNA gene, partial sequence & 101 & 100 & $2 e-39$ & 97 & KR399992.1 \\
\hline A6 & Lactococcus garvieae strain RCB131 $16 \mathrm{~S}$ ribosomal RNA gene, partial sequence & 80 & 100 & $8 e-33$ & 100 & KT260343.1 \\
\hline A7 & Enterococcus durans strain R08-28 $16 \mathrm{~S}$ ribosomal RNA gene, partial sequence & 44 & 100 & $1 e-14$ & 100 & JF896442.1 \\
\hline A8 & Enterococcus faecium strain 133165 ribosomal RNA gene, partial sequence & 166 & 98 & $5 e-54$ & 91 & EU418442.1 \\
\hline A9 & Enterococcus faecium strain SA-10 $16 \mathrm{~S}$ ribosomal RNA gene, partial sequence & 574 & 100 & 0.0 & 100 & KR265372.1 \\
\hline A10 & Enterococcus faecium strain UW7606×64/3 TC1, complete genome & 354 & 100 & 0.0 & 99 & CP013009.1 \\
\hline A11 & Lactococcus garvieae strain RCB99 $16 \mathrm{~S}$ ribosomal RNA gene, partial sequenc & 405 & 100 & 0.0 & 100 & KT260311.1 \\
\hline A12 & Lactococcus garvieae strain RCB130 16S ribosomal RNA gene, partial sequence & 96 & 98 & $5 e-31$ & 94 & KT260343.1 \\
\hline
\end{tabular}

BLAST, Basic Local Alignment Search Tool; RNA, ribosomal nucleic acid.

a clear halo was observed, indicating the presence of a polysaccharide capsule.

\section{Genotypic characterisation of exopolysaccharides}

The amplification of the EPS biosynthesis gene cluster by long-range PCR was attempted as described by Miyauchi et al. (2012). Figure 3 shows a product of $\sim 750$ bp for all isolates tested, consistent with the product sizes observed in the $\mathrm{KG}^{+}$ (non-capsulated) strains tested in the study by Miyauchi et al. (2012). The presence of insertion sequences (ISs) flanking the EPS biosynthesis gene cluster in the $\mathrm{KG}^{-}$strain $\mathrm{Lg} 2$ used in the study by Miyauchi et al. (2012) may suggest that the EPS biosynthesis gene cluster could be inserted at different genomic loci in strains other than $\mathrm{Lg} 2$. If this should happen, the primers used by Miyauchi et al. (2012) will fail to amplify this gene cluster in other strains as these primers flank the gene cluster including the ISs. Therefore, primers that amplify the first gene in the cluster, a transcriptional regulator (epsR), were designed. However, PCR amplification of this gene yielded no product in any isolates tested.

\section{Detection of extracellular proteins}

Extracellular proteins of eight L. garvieae strains, purified and concentrated by Amicon ${ }^{\circledR}$ Ultra-4 10K Centrifugal Filter Units (Merck), were visualised on a $12 \%$ SDS-PAGE gel. More protein bands were observed in strain A3 in comparison to other strains, as can be seen in Figure 4 . No clear bands were observed in the negative control, which consisted of sterile TSB medium, indicating that the growth medium did not contribute any of the protein bands detected and identified in supernatants (Table 4).

\section{Detection of putative virulence factor genes by polymerase chain reaction}

Visualisation of PCR products by gel electrophoresis, as presented in Figure 5, followed by sequencing and nucleotide database search confirmed the presence of seven candidate virulence genes in genomes of seven South African isolates as well as an avirulent reference strain, NCFB657.

\section{Discussion}

All putative virulence genes under investigation were present in the genomes of all South African isolates used in this study, as well as the reference strain NCFB657. These results are in accordance with the findings of Türe and Altinok (2016), who reported the presence of this set of putative virulence factor genes in the genomes of a total of 34 L. garvieae strains, including the avirulent reference strains ATCC $^{\circledR} 49156$ and ATCC $^{\circledR} 4392$ and isolates from diseased rainbow trout in Turkey, France, Iran, Spain and Italy.

No phenotypic proof of the presence of a polysaccharide capsule was collected in this study using light microscopy. These observations alone, however, should not be considered definitive proof of the absence of capsules, as Yoshida et al. (1997) reported that neither negative staining using Muir's method or Indian ink, nor the Quellung reaction was successful in visualising L. garvieae $\mathrm{KG}^{-}$capsules.

The EPS capsule influences a variety of host interactions, including adhesion, invasion and sensitivity to opsonophagocytosis, and is a well-recognised virulence factor of L. garvieae. The eps locus of L. garvieae Lg2 was reported to be a genomic island, attributed to the presence of ISs IS982 on both ends of the EPS biosynthesis cluster, differences in guanine-cytosine (GC) content between the Lg2 chromosomal average and cluster (39\% and 31\%, respectively) and demonstration that this locus was probably inserted into the locus syntenic to four sequenced $L$. lactis strains (Morita et al. 2011). It was postulated that this gene cluster might have been present in the avirulent noncapsulated reference strain ATCC $^{\circledR} 49156$, originally isolated from diseased yellowtail, and was lost during repeated subculturing (Morita et al. 2011). The negative results obtained using the PCR amplification of the EPS biosynthesis cluster in this study may reflect a similar phenomenon as the attenuation of ATCC $^{\circledR} 49156$ by repeated subculturing. The strains used in this study were isolated from diseased rainbow trout in 1986 and has been repeatedly subcultured under laboratory conditions, which may be selective for the non-capsulated phenotype as no selective pressure in the form of host immune mechanisms is applied. Presence of the capsule confers fitness only when growth occurs under in vivo conditions. Therefore, deletion of the EPS biosynthesis cluster, and subsequently the loss capsulated $\mathrm{KG}^{-}$phenotype, is a possible explanation for the results obtained. However, in light of recent findings by Türe and Altinok (2016), the 

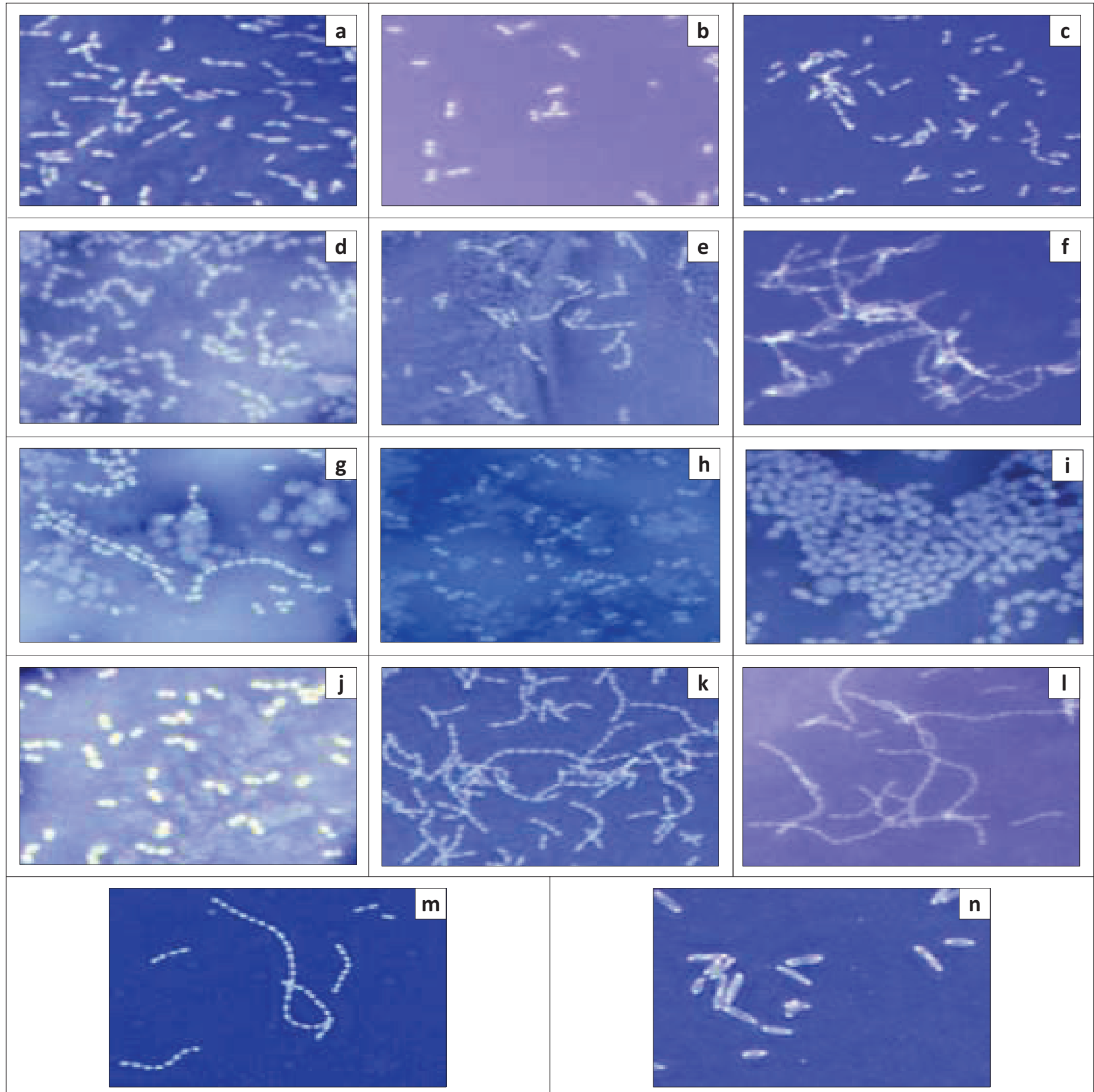

FIGURE 2: Negative staining of Lactococcus garvieae A1-A12 using nigrosin, visualised using 100x magnification. L garvieae NCFB657 and Pseudomonas aeruginosa were included as negative and positive controls, respectively. (a), A1; (b), A2; (c), A3, (d), A4; (e), A5; (f), A6; (g), A7; (h), A8; (i), A9; (j), A10; (k), A11; (l), A12; (m), L. garvieae NCFB657; (n), P. aeruginosa.

isolates investigated in this study may not have been capsulated at the time of isolation.

Previous immunoproteomic studies on L. garvieae did not identify enolase, $60 \mathrm{kDa}$ chaperonin or phosphoenolpyruvateprotein phosphotransferase as antigens recognised by rabbit antisera (Shin et al. 2007) or olive flounder (Paralichthys olivaceus) antisera (Shin et al. 2009). However, L-lactate dehydrogenase was reported as an antigen common to both $\mathrm{KG}^{+}$and $\mathrm{KG}^{-}$serotypes (Shin et al. 2009). Although enolase, $60 \mathrm{kDa}$ chaperonin and phosphoenolpyruvate-protein phosphotransferase have not been identified as antigens in
L. garvieae, the identification of these extracellular proteins is of interest as they have been described as moonlighting proteins with involvement in virulence and regulation of virulence genes in Gram-positive bacteria.

Enolase (EC 4.2.1.11) is a glycolytic enzyme responsible for catalysing the conversion of 2-phosphoglycerate to phosphoenolpyruvate in the second last step of glycolysis, and the reverse reaction in gluconeogenesis (Lee et al. 2006). Additionally, this enzyme plays a role in virulence of Gram-positive and Gram-negative bacteria (Bergmann et al. 2001; Nogueira et al. 2013; Pancholi \& Fischetti 1998). 


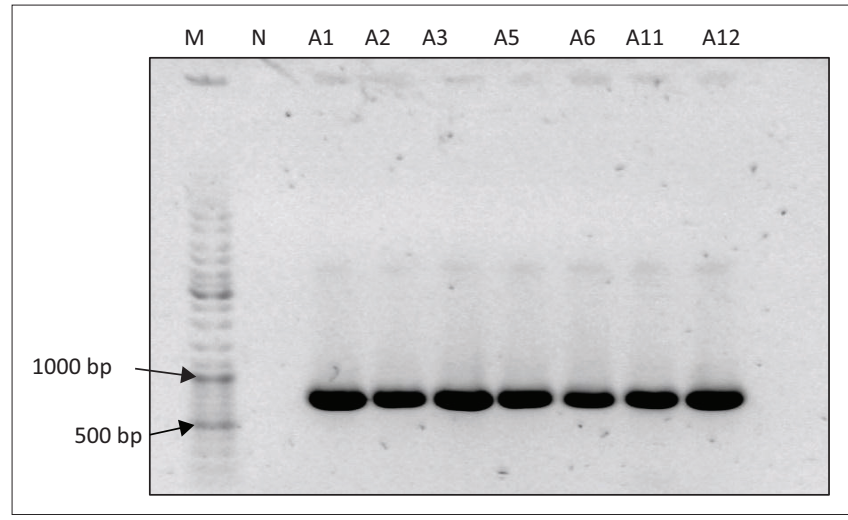

bp, base pair.

LR PCR, long range polymerase chain reaction.

FIGURE 3: Visualisation of LR PCR products on a $1 \%$ agarose gel. A band of $\sim 750 \mathrm{bp}$ was observed in all isolates tested, indicating the absence of an exopolysaccharide gene cluster in the genomes of these isolates.

TABLE 4: Top protein scores generated by query of the SWISS-PROT protein sequence database with the search engine Mascot, following nano liquid chromatography-tandem mass spectrometry analysis of extracellular proteins from Lactococcus garvieae strain A3.

\begin{tabular}{llcc}
\hline Band no. & Top protein scores & $\begin{array}{c}\text { MOWSE score } \\
(\boldsymbol{p}<\mathbf{0 . 0 5})\end{array}$ & MW (kDa) \\
\hline 1 & $\begin{array}{l}\text { L-lactate dehydrogenase } \\
\text { OS=Lactobacillus helveticus }\end{array}$ & 174 & 35,089 \\
2 & $\begin{array}{l}\text { Enolase OS=Streptococcus suis (strain } \\
\text { 98HAH33) }\end{array}$ & 234 & 47,066 \\
3 & $\begin{array}{l}\text { 60 kDa chaperonin OS=Lactobacillus } \\
\text { brevis (strain ATCC }\end{array}$ & 92 & 57,044 \\
4 & $\begin{array}{l}\text { Phosphoenolpyruvate-protein } \\
\text { phosphotransferase OS=Lactococcus } \\
\text { lactis subsp. lactis (strain IL1403) }\end{array}$ & 137 & 62,521 \\
\hline
\end{tabular}

MOWSE, molecular weight search

$\mathrm{MW}$, molecular weight; $\mathrm{kDa}$, kilodalton

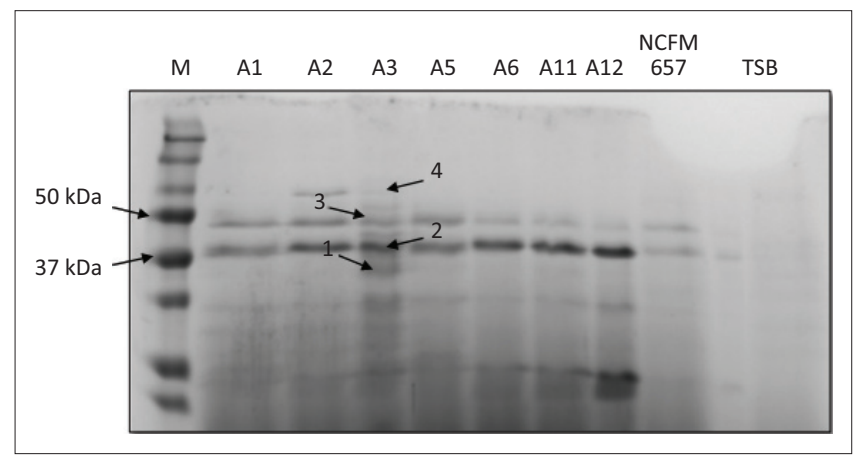

$\mathrm{M}$, molecular weight marker (in $\mathrm{kDa}$ ).

FIGURE 4: Extracellular proteins of eight Lactococcus garvieae strains, including the reference strain NCFB657, visualised on a $12 \%$ sodium dodecyl sulphatepolyacrylamide gel electrophoresis gel. A negative control (sterile tryptic soy broth (TSB)) was included.

Numerous studies on the moonlighting activities of enolase in prokaryotes have highlighted its involvement in binding of host plasmin(ogen), a process of critical importance to invasion of host tissues (Bergmann et al. 2001; Henderson \& Martin 2011; Pancholi \& Fischetti 1998). Binding of host plasmin(ogen) to its surface endows the bacterial cell with protease activity, which facilitates invasion and dissemination in the host (Winram \& Lottenberg 1996). Surface-associated enolase with plasmin(ogen) binding activity has been reported in human streptococcal pathogens Streptococcus pyogenes (Pancholi \& Fischetti 1998), Streptococcus pneumoniae (Bergmann et al. 2001) and piscine pathogen Streptococcus iniae (Kim et al. 2007).
A study by Wang et al. (2015) confirmed the immunogenicity of $S$. iniae recombinant $\alpha$-enolase in mice, showing that immunisation with $\alpha$-enolase elicited a significant increase in specific immunoglobulin $G$ in comparison with the control group and ultimately protected mice against systemic $S$. iniae infection (Wang et al. 2015). The ability of S. iniae $\alpha$-enolase to confer protection in Nile tilapia (LaFrentz, Shoemaker \& Klesius 2011) and turbot (Scophthalmus maximus) (Zhang, Zhang \& Sun 2014) has been previously suggested.

The prokaryotic $60 \mathrm{kDa}$ chaperonins (Cpn60) is a highly conserved group of proteins that mediate intracellular protein folding to ensure correct functioning (Henderson, Fares \& Lund 2013). Cpn60 is found on the cell surface of a variety of bacteria where it functions mainly as an adhesin. Among the members of the order Lactobacillales reported to produce extracellular Cpn60 are Lactobacillus johnsonii (Bergonzelli et al. 2006), L. lactis (Katakura et al. 2010), Streptococcus agalactiae (Hughes et al. 2002) and Streptococcus suis (Wu, Zhang \& Lu 2008). Diverse ligands of bacterial cellsurface Cpn60 have been identified, ranging from mucin, lactoferrin, invertase and glycosphingolipids to integrin receptors CD11/CD18, $\alpha_{\mathrm{v}} \beta_{3}$ and dendritic cell-specific intercellular adhesion molecule-3-grabbing non-integrin (Henderson et al. 2013). Proteomic analysis of S. iniae ATCC ${ }^{\circledR}$ 29178 and L. garvieae KG9408 (capsulated) by 2-dimensional gel electrophoresis identified Cpn60 in both fish pathogens (Shin et al. 2006), but subsequent immunoproteomic analyses by immunoblotting with rabbit and olive flounder antiserum did not identify this protein as an antigen of either capsulated or non-capsulated L. garvieae (Shin et al. 2007, 2009).

Phosphoenolpyruvate (PEP)-protein phosphotransferase (EC 2.7.3.9) is a key enzyme in the bacterial phosphotransferase system (PTS) (Deutscher et al. 2014). The multifunctionality of S. pneumoniae PEP-protein phosphotransferase (PtsA) has been investigated by demonstrating its presence on the cell surface of $S$. pneumoniae using immunofluorescence techniques and using recombinantly expressed PtsA (rPtsA) to screen a filamentous phage display library to identify peptides capable of inhibiting adhesion of S. pneumoniae to human lung adenocarcinoma cells (Mizrachi Nebenzahl et al. 2016). These peptides showed homology to various human ECM proteins, including multimerin I, protocadherin 19 and collagen type VII 1 , suggesting that PtsA acts as an adhesin of S. pneumoniae (Mizrachi Nebenzahl et al. 2016). Furthermore, rPtsA was identified as a candidate target for vaccine development by showing that immunisation of mice with rPtsA offered protection against pneumococcal challenge via different routes (Mizrachi Nebenzahl et al. 2016).

Pore-forming toxins are secreted by a variety of Gramnegative and Gram-positive pathogenic bacteria to disrupt lipid bilayers of host cells (Iacovache, van der Goot \& Pernot 2008). The putative virulence gene hly1 was reported to display $56 \%$ amino acid sequence homology to a protein in E. faecalis containing motif PF03006 conserved in proteins with haemolytic activity (Morita et al. 2011). Proteins containing this motif are classified in the haemolysin-III 


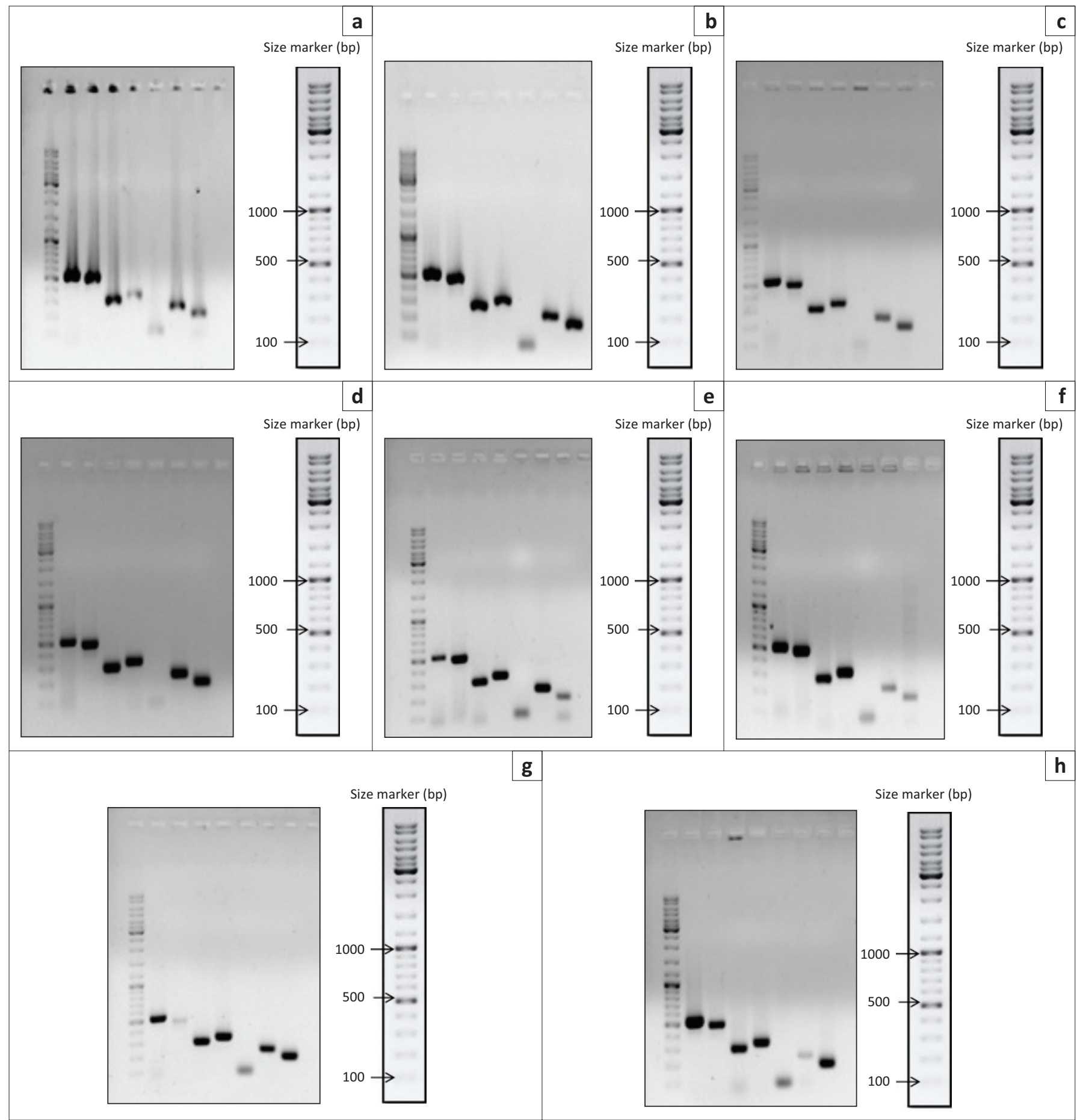

bp, base pair.

FIGURE 5: Putative virulence factor genes were detected by polymerase chain reaction assays. Products are shown visualised on $1 \%$ agarose gels. The first lanes on all gels are size markers, and the subsequent lanes represent virulence genes in the following order: hly1; hly2; hly3; nox; sod; pavA; psaA. (a), A1; (b), A2; (c), A3, (d), A5; (e), A6; (f), A11; (g), A12; (h), NCFB657.

related family (http://pfam.xfam.org/; Finn et al. 2014). Based on the structural features of $h l y 1$, it is classified as a member of the $\alpha$-pore-forming toxins, which include colicins of Escherichia coli (Iacovache et al. 2008). Another putative haemolysin under investigation in this study, encoded by hly2, was reported to display $72 \%$ amino acid homology to with a haemolysin in S. suis (Morita et al. 2011). The gene product of hly3 was reported to display 59\% amino acid sequence similarity to a haemolysin in S. pyogenes (Morita et al. 2011), an organism associated with $\beta$-haemolytic activity.
Reactive oxygen species (ROS) have various noxious effects on cells (Yesilkaya et al. 2000). Protection against oxidative stress is afforded by the enzymatic removal of ROS by catalase and SOD. The LAB are catalase negative (Collins et al. 1983); therefore, SOD (EC 1.15.1.1) represents a major defence mechanism against oxidative stress in L. garvieae. The detoxifying properties of SOD can also enhance intraphagocytic survival of bacteria upon generation of bactericidal ROS as a result of respiratory burst (Fang et al. 2015). The enzyme NOX reduces molecular oxygen to 
$\mathrm{H}_{2} \mathrm{O}$ or $\mathrm{H}_{2} \mathrm{O}_{2}$ and was proposed to perform an important role in defence against $\mathrm{O}_{2}$ toxicity and $\mathrm{O}_{2}$ sensing, which are essential functions during aerobic growth of LAB (Muchnik et al. 2013). The importance of NOX in the virulence of S. pneumoniae has been established by Yu et al. (2001) by demonstrating significant attenuation of a nox-deficient mutant, leading to the conclusion that NOX is required for in vivo proliferation in $\mathrm{O}_{2}$-rich environments. Random peptide library phage display was used to identify several extracellular matrix proteins as ligands to recombinant NOX, suggesting that the enzyme may play a multifunctional role in virulence by additionally acting as an adhesin (Muchnik et al. 2013).

Adherence to host cells is a prerequisite step in the colonisation processes of pathogenic and commensal microbes (Kline et al. 2009). Morita et al. (2011) reported that protein LCGL_1330 of L. garvieae Lg2 shared $62 \%$ amino acid identity with S. pneumoniae TIGR4 PavA and contained a fibronectin-binding motif (PF05833). Pneumococcal adherence and virulence factor A (PavA) is a cell surface-localised fibronectin-binding protein that lacks both typical signal sequences for secretion as well as the LPXTG anchorage motif characteristic of cell surface proteins (Holmes et al. 2001). Another protein with amino acid sequence homology (49\%) to a S. pneumoniae cell surface-exposed virulence determinant, pneumococcal surface antigen A (PsaA), was identified in Lg2 by Morita et al. (2011). This protein was originally identified as a putative adhesin (Berry \& Paton 1996); however, its structure was not found to be consistent with the function of adhesion (Johnston et al. 2004). Its function was revealed to be a divalent metal ionbinding lipoprotein component of an ATP-binding cassette (ABC) transporter for $\mathrm{Mn}^{2+}$ (Johnston et al. 2004).

\section{Conclusion}

The major virulence factor of L. garvieae defined thus far is the antiphagocytic polysaccharide capsule. With the aid of next-generation sequencing of full genomes, several other putative virulence factors have been identified. Despite rapid advances in the areas of molecular biology and bioinformatics, its pathogenic processes are inadequately understood. No polysaccharide capsule was detected in any of the South African strains studied. Putative virulence factors with roles in adhesion, cytolytic activity, oxidative stress tolerance and metal homeostasis were identified in a set of seven South African fish pathogenic L. garvieae isolates, as well as an avirulent isolate. Detection of extracellular proteins of South African fish pathogenic L. garvieae isolates in this study identified candidates that may be investigated as subunit vaccines in future.

Specific virulence factors responsible for the pathogenicity of L. garvieae could not be identified, as putative virulence factor genes were present in both the fish pathogenic isolates and the avirulent isolate. All virulence factors discussed in this study, with the exception of haemolysins, are virulence lifestyle factors that indirectly contribute to host damage. These virulence lifestyle factors aid in the infection process by evasion of the host's innate immune system, cofactor homeostasis, systemic invasion and dissemination in the host and adhesion to host tissues. Future studies should focus on investigating differential expression of virulence lifestyle and true virulence genes during growth in the host environment versus laboratory conditions.

\section{Acknowledgements Competing interests}

The authors declare that they have no financial or personal relationships that may have inappropriately influenced them in writing this article.

\section{Authors' contributions}

R.R.B. and C.E.B. were the project leaders and responsible for the project design, while C.M.M. performed all experiments and wrote the manuscript.

\section{References}

Aguado-Urda, M., Gibello, M., Mar Blanco, M., López-Campos, G., Cutuli, M.T. \& Fernández-Garayzábal, J.F., 2012, 'Characterization of plasmids in a human clinical strain of Lactococcus garvieae', PLoS One 7(6). https://doi.org/10.1371/journal. pone.0040119

Aoki, T., Takami, K. \& Kitao, T., 1990, 'Drug resistance in a nonhemolytic Streptococcus sp. isolated from cultured yellowtail Seriola quinqueradiata', Diseases of Aquatic Organisms 8, 171-177.

Altschul, S.F., Gish, W., Miller, W., Myers, E.W. \& Lipman, D.J., 1990, 'Basic local alignment search tool', Journal of Molecular Biology 2015(September), 403-410.

Austin, B. \& Austin, A.D., 2012, Bacterial fish pathogens: Diseases of farmed and wild fish, 5th edn., Springer, Chichester, UK.

Bateman, A., Martin, M.J., O’Donovan, C., Magrane, M., Apweiler, R., Alpi, E. et al., 2015, 'UniProt: A hub for protein information', Nucleic Acids Research 43(D1), D204-D212. https://doi.org/10.1093/nar/gku989

Bekker, A., Hugo, C., Albertyn, J., Boucher, C.E. \& Bragg, R.R., 2011, 'Pathogenic Grampositive cocci in South African rainbow trout, Oncorhynchus mykiss (Walbaum)', Journal of Fish Diseases 34(6), 483-487. https://doi.org/10.1111/j.13652761.2011.01259.x

Bercovier, H., Ghittino, C. \& Eldar, A., 1997, 'Immunization with bacterial antigens: Infections with streptococci and related organisms', Developments in Biological Standardization 90, 153-160.

Bergmann, S., Rohde, M., Chhatwal, G.S. \& Hammerschmidt, S., 2001, ' $\alpha$-Enolase of Streptococcus pneumoniae is a plasmin(ogen)-binding protein displayed on the bacterial cell surface', Molecular Microbiology 40(6), 1273-1287. https://doi. org/10.1046/j.1365-2958.2001.02448.x

Bergonzelli, G.E. Granato, D., Pridmore, R.D., Marvin-Guy, L.F., Donnicola, D. \& Corthésy-Theulaz, I.E., 2006, 'GroEL of Lactobacillus johnsoni La1 (NCC533) is cell surface associated: Potential role in interactions with the host and the gastric surface associated: Potential role in interactions with the host and the gastric
pathogen Helicobacter pylori', Infection and Immunity 74(1), 425-434. https:// pathogen Helicobacter pylori',
doi.org/10.1128/IAI.74.1.425

Berry, A.M. \& Paton, J.C., 1996, 'Sequence heterogeneity of PsaA, a 37-kilodalton putative adhesin essential for virulence of Streptococcus pneumoniae', Infection and Immunity 64(12), 5255-5262.

Beumer, A. \& Robinson, J.B., 2005, 'A broad-host-range, generalized transducing phage (SN-T) acquires $16 \mathrm{~S}$ rRNA genes from different genera of bacteria', American Society for Microbiology 71(12), 8301-8304. https://doi.org/10.1128/AEM.71.12.8301

Bragg, R.R. \& Broere, J.S., 1986, 'Streptococcosis in rainbow trout in South Africa', Fish Pathology 6(3), 89 .

Collins, M.D., Farrow, J.A., Phillips, B.A. \& Kandler, O., 1983, 'Streptococcus garvieae sp. nov. and Streptococcus plantarum sp. nov.', Journal of General Microbiology 129, 3427-3431. https://doi.org/10.1099/00221287-129-11-3427

Deutscher, J., Aké, F.M., Derkaoui, M., Zébré, A.C., Cao, T.N., Bouraoui, H. et al., 2014, 'The bacterial phosphoenolpyruvate: Carbohydrate phosphotransferase system: Regulation by protein phosphorylation and phosphorylation-dependent proteinprotein interactions', Microbiology and Molecular Biology Reviews: MMBR 78(2), 231-56. https://doi.org/10.1128/MMBR.00001-14

Eldar, A., Ghittino, C., Asanta, L., Bozzetta, E., Goria, M., Prearo, M. et al., 1996, 'Enterococcus seriolicida is a junior synonym of Lactococcus garvieae, a causative agent of septicemia and meningoencephalitis in fish', Current Microbiology 32(2), 85-88. https://doi.org/10.1007/s002849900015

Fang, L., Shen, H., Tang, Y. \& Fang, W., 2015, 'Superoxide dismutase of Streptococcus suis serotype 2 plays a role in anti-autophagic response by scavenging reactive oxygen species in infected macrophages', Veterinary Microbiology 176(3-4), 328-336. https://doi.org/10.1016/j.vetmic.2015.02.006 
Finn, R.D., Bateman, A., Clements, J., Coggill, P., Eberhardt, R.Y., Eddy, S.R. et al., 2014, 'Pfam: The protein families database', Nucleic Acids Research 42(D1), 222-230. 'Pfam: The protein families database',
https://doi.org/10.1093/nar/gkt1223

Gibello, A., Galán-Sánchez, F., Blanco, M.M., Rodríguez-Iglesias, M., Domínguez, L. \& Fernández-Garayzábal, J.F., 2016, 'The zoonotic potential of Lactococcus garviea: An overview on microbiology, epidemiology, virulence factors and relationship with its presence in foods', Research in Veterinary Science 109, 59-70. https://doi. org/10.1016/j.rvsc.2016.09.010

Henderson, B., Fares, M.A. \& Lund, P.A., 2013, 'Chaperonin 60: A paradoxical, evolutionarily conserved protein family with multiple moonlighting functions', Biological Reviews 88(4), 955-987. https://doi.org/10.1111/brv.12037

Henderson, B. \& Martin, A., 2011, 'Bacterial virulence in the moonlight: Multitasking bacterial moonlighting proteins are virulence determinants in infectious disease Infection and Immunity 79(9), 3476-3491. https://doi.org/10.1128/IAI.00179-11

Heuer, O.E., Kruse, H., Grave, K., Collignon, P., Karunasagar, I. \& Angulo, F.J., 2009 'Human health consequences of use of antimicrobial agents in aquaculture' Clinical Infectious Diseases 49, 1248-1253. https://doi.org/10.1046/10.1086/ 605667

Holmes, A.R., McNab, R., Millsap, K.W., Rohde, M., Hammerschmidt, S., Mawdsley, J.L. et al., 2001, 'The pavA gene of Streptococcus pneumoniae encodes a fibronectin-binding protein that is essential for virulence', Molecular Microbiology 41, 1395-1408. https://doi.org/10.1046/j.1365-2958.2001.02610.x

Hughes, M.J.G., Moore, J.C., Lane, J.D., Wilson, R., Pribul, P.K., Younes, Z.N. et al., 2002 , 'Identification of major outer surface proteins of Streptococcus agalactiae', Infection and Immnunity 70(3), 1254-1259. https://doi.org/10.1128/IAI.70.3.1254

lacovache, I., van der Goot, F.G. \& Pernot, L., 2008, 'Pore formation: An ancient yet complex form of attack', Biochimica et Biophysica Acta 1778, 1611-1623. https:// doi.org/10.1016/j.bbamem.2008.01.026

Johnston, J., Myers, L.E., Ochs, M.M., Benjamin, W.H. Jr, Briles, D.E. \& Hollingshead, S.K., 2004, 'Lipoprotein PsaA in virulence of Streptococcus pneumoniae: Surface accessibility and role in protection from superoxide', Infection and Immunity accessibility and role in protection from superoxide', Infection and
72(10), 5858-5867. https://doi.org/10.1128/IAl.72.10.5858-5867.2004

Katakura, Y., Sano, R., Hashimoto, T., Ninomiya, K. \& Shioya, S., 2010, 'Lactic acid bacteria display on the cell surface cytosolic proteins that recognize yeast mannan', Applied Microbiology and Biotechnology 86(1), 319-326. https://doi. mannan', Applied Microbiology
org/10.1007/s00253-009-2295-y

Kearse, M., Moir, R., Wilson, A., Stones-Havas, S., Cheung, M., Sturrock, S. et al., 2012, 'Geneious Basic: An integrated and extendable desktop software platform for the organization and analysis of sequence data', Bioinformatics 28(12), 1647-1649. https://doi.org/10.1093/bioinformatics/bts199

Kim, M.S., Choi, S.H., Lee, E.H., Nam, Y.K., Kim, S.K. \& Kim, K.H., 2007, ' $\alpha$-enolase, a plasmin(ogen) binding and cell wall associating protein from a fish pathogenic Streptococcus iniae strain', Aquaculture 265(1-4), 55-60. https://doi.org/ 10.1016/j.aquaculture.2007.01.034

Kline, K.A., Fälker, S., Dahlberg, S., Normark, S. \& Henriques-Normark, B., 2009 'Bacterial adhesins in host-microbe interactions', Cell Host and Microbe $5(6)$ 580-592. https://doi.org/10.1016/j.chom.2009.05.011

Kusuda, R., Kawai, K., Salati, F., Banner, C.R. \& Fryer, J.L., 1991, 'Enterococcus seriolicida $\mathrm{sp}$. nov., a fish pathogen', International Journal of Evolutionary Bacteriology 41(July), 406-409.

Kusuda, R. \& Hamaguchi, M., 1988, 'Extracellular and intracellular toxins of Streptococcus sp. isolated from yellowtail', Bulletin of the European Association of Fish Pathologists 8, 9-10. https://doi.org/10.1099/00207713-41-3-406

LaFrentz, B.R., Shoemaker, C.A. \& Klesius, P.H., 2011, 'Immunoproteomic analysis of the antibody response obtained in Nile tilapia following vaccination with a
Streptococcus iniae vaccine', Veterinary Microbiology $152(3-4), 346-352$. https:// Streptococcus iniae vaccine', Veterinary
doi.org/10.1016/j.vetmic.2011.04.033

Lee, J.-H., Kang, H.K., Moon, Y.H., Cho, D.L., Kim, D., Choe, J.Y. et al., 2006, 'Cloning, expression and characterization of an extracellular enolase from Leuconostoc mesenteroides', FEMS Microbiology Letters 259(2), 240-248. https://doi.org/ mesenteroides', FEMS Microbiology
10.1111/j.1574-6968.2006.00274.x

Miyauchi, E., Toh, H., Nakano, A., Tanabe, S. \& Morita, H., 2012, 'Comparative genomic analysis of Lactococcus garvieae strains isolated from different sources reveals candidate virulence genes', International Journal of Microbiology 2012, 728276 https://doi.org/10.1155/2012/728276

Mizrachi Nebenzahl, Y., Blau, K., Kushnir, T., Shagan, M., Portnoi, M., Cohen, A. et al., 2016, 'Streptococcus pneumoniae cell-wall-localized phosphoenolpyruvate protein phosphotransferase can function as an adhesin: Identification of its host target molecules and evaluation of its potential as a vaccine', PLoS One 11(3), e0150320. https://doi.org/10.1371/journal.pone.0150320

Morita, H., Toh, H., Oshima, K., Yoshizaki, M., Kawanishi, M., Nakaya, K. et al., 2011, 'Complete genome sequence and comparative analysis of the fish pathogen Lactococcus garvieae', PLoS One 6(8), 1-8. https://doi.org/10.1371/journal. pone.0023184

Muchnik, L., Adawi, A., Ohayon, A., Dotan, S., Malka, I., Azriel, S. et al., 2013, 'NADH oxidase functions as an adhesin in Streptococcus pneumoniae and elicits protective immune response in mice', PLoS One 8(4), 13p. https://doi.org/10.1371/ journal.pone.0061128
Nogueira, S.V., Backstedt, B.T., Smith, A.A., Qin, J.H., Wunder, E.A., Ko, A. et al., 2013, 'Leptospira interrogans enolase is secreted extracellularly and interacts with plasminogen', PLoS One 8(10), 1-11. https://doi.org/10.1371/journal.pone. 0078150

Pancholi, V. \& Fischetti, V.A., 1998, ' $\alpha$-Enolase, a novel strong plasmin(ogen) binding protein on the surface of pathogenic streptococci', The Journal of Biological Chemistry 273(23), 14503-14515. https://doi.org/10.1074/jbc.273.23.14503

Perkins, D., Pappin, D.J., Creasy, D.M. \& Cottrell, J.S., 1999, 'Probability-based protein identification by searching sequence databases using mass spectrometry data', Electrophoresis 20(18), 3551-3567. https://doi.org/10.1002/(sici)1522-2683 (19991201)20:18<3551::aid-elps3551>3.0.co;2-2

Ravelo, C., Magariños, B., Romalde, J.L. \& Toranzo, A.E., 2001, 'Conventional versus miniaturized systems for the phenotypic characterization of Lactococcus garvieae strains', Bulletin of the European Association of Fish Pathologists 21(4), 136-144.

Romero, J., Feijoo, C. \& Navarrete, P., 2012, 'Antibiotics in aquaculture - Use, abuse and alternatives, health and environment in aquaculture', in Health and environment in aquaculture [online], IntechOpen, pp. 160-198, viewed 20 August 2016, from https://www.intechopen.com/books/health-and-environment-in-aquaculture/ antibiotics-in-aquaculture-use-abuse-and-alternatives.

Sambrook, J. \& Russell, D.W., 2001, Molecular cloning: A laboratory manual, 3rd edn., J. Inglis, A. Boyle \& A. Gann (eds.), Cold Spring Harbor Laboratory Press, New York.

Shin, G.W., Palaksha, K.J., Yang, H.H., Shin, Y.S., Kim, Y.R., Lee, E.Y. et al., 2006, 'Partial two-dimensional gel electrophoresis (2-DE) maps of Streptococcus iniae ATCC29178 and Lactococcus garvieae KG9408', Diseases of Aquatic Organisms 70(1-2), 71-79. https://doi.org/10.3354/dao070071

Shin, G.W., Palaksha, K.J., Kim, Y.R., Nho, S.W., Cho, J.H., Heo, N.E. et al., 2007 'Immunoproteomic analysis of capsulate and non-capsulate strains of Lactococcus 'Immurvieae', Veterinary Microbiology 119(2-4), 205-212. https://doi.org/10.1016/j. garvieae', Veterinary
vetmic.2006.08.021

Shin, G.W., Nho, S.W., Park, S.B., Jang, H.B., Cha, I.S., Ha, M.A. et al., 2009, 'Comparison of antigenic proteins from Lactococcus garvieae KG (-) and KG (+) strains that are recognized by olive flounder (Paralichthys olivaceus) antibodies', Veterinary recognized by olive flounder (Paralichthys olivaceus) antibodies, Veterinary
Microbiology 139(1-2), 113-120. https://doi.org/10.1016/j.vetmic.2009.05.007

Teixeira, L.M., Merquior, V.L., Vianni, M.C., Carvalho, M.G., Fracalanzza, S.E., Steigerwalt, A.G. et al., 1996, 'Phenotypic and genotypic characterization of atypical Lactococcus garvieae strains isolated from water buffalos with subclinical mastitis and confirmation of $L$. garvieae as a senior subjective synonym of Enterococcus seriolicida', International Journal of Systematic Bacteriology 46(3), Enterococcus seriolicida', International Journal of Systema

Thanh, V.N., 2006, 'Lipomyces orientalis sp. nov., a yeast species isolated from soil in Vietnam', International Journal of Systematic and Evolutionary Microbiology 56(8), 2009-2013. https://doi.org/10.1099/ijs.0.64241-0

Türe, M., Haliloğlu, H.i., Altuntas, C., Boran, H. \& Kutlu, i.., 2014, 'Comparison of experimental susceptibility of rainbow trout (Oncorhynchus mykiss), turbot (Psetta maxima), black sea trout (Salmo trutta labrax) and sea bass (Dicentrarchus labrax) to Lactococcus garvieae', Turkish Journal of Fisheries and Aquatic Science 14, 507-513. https://doi.org/10.4194/1303-2712-v14_2_22

Türe, M. \& Altinok, I., 2016, 'Detection of putative virulence genes of Lactococcus garvieae', Diseases of Aquatic Organisms 119(1), 59-66. https://doi.org/10.3354/ dao02981

Wang, J., Wang, K., Chen, D., Geng, Y., Huang, X., He, Y. et al., 2015, 'Cloning and characterization of surface-localized alpha-enolase of Streptococcus iniae, an effective protective antigen in mice', International Journal of Molecular
Sciences 16(7), 14490-14510. https://doi.org/10.3390/ijms160714490 \rijms 160714490 [pii] Winram, S.B. \& Lottenberg, R., 1996, 'The plasmin-binding protein Plr of group
A streptococci is identified as glyceraldehyde-3-phosphate dehydrogenase', Microbiology 142(8), 2311-2320. https://doi.org/10.1099/13500872-142-8-2311

Wong, C., Sridhara, S., Bardwell, J.C. \& Jakob, U., 2000, 'Heating greatly speeds Coomassie blue staining and destaining', BioTechniques 28(3), 426-432.

Wu, Z., Zhang, W. \& Lu, C., 2008, 'Immunoproteomic assay of surface proteins of Streptococcus suis serotype 9 ', FEMS Immunology and Medical Microbiology 53(1), 52-59. https://doi.org/10.1111/j.1574-695X.2008.00401.x

Yesilkaya, H., Kadioglu, A., Gingles, N., Alexander, J.E., Mitchell, T.J. \& Andrew, P.W., 2000, 'Role of manganese-containing superoxide dismutase in oxidative stress and Role of manganese-containing superoxide dismutase in oxidative stress and
virulence of Streptococcus pneumoniae', Infection and Immunity 68(5), 2819-2826. virulence of Streptococcus pneumoniae', Infection
https://doi.org/10.1128/IAl.68.5.2819-2826.2000

Yoshida, T., Endo, M., Sakai, M. \& Inglis, V., 1997, 'A cell capsule with possible involvement in resistance to opsonophagocytosis in Enterococcus seriolicida isolated from yellowtail Seriola quinqueradiata', Disease of Aquatic Organisms 29(3), 233-235. https://doi.org/10.3354/dao029233

Yu, J., Bryant, A.P., Marra, A., Lonetto, M.A., Ingraham, K.A., Chalker, A.F. et al., 2001, 'Characterization of the Streptococcus pneumoniae NADH oxidase that is required for infection', Microbiology 147(2001), 431-438. https://doi.org/10.1099/ 00221287-147-2-431

Zhang, B.C., Zhang, J. \& Sun, L., 2014, 'Streptococcus iniae SF1: Complete genome sequence, proteomic profile, and immunoprotective antigens', PLoS One 9(3), e91324. https://doi.org/10.1371/journal.pone.0091324 